\title{
TTR
}

Traduction, terminologie, rédaction

\section{Freddie Plassard. Lire pour traduire. Paris, Les Presses de la Sorbonne Nouvelle, 2007, 323 p.}

\section{Marco A. Fiola}

Volume 20, numéro 2, 2e semestre 2007

TTR a 20 ans II

TTR Turns 20 II

URI : https://id.erudit.org/iderudit/018829ar

DOI : https://doi.org/10.7202/018829ar

Aller au sommaire du numéro

\section{Éditeur(s)}

Association canadienne de traductologie

ISSN

0835-8443 (imprimé)

1708-2188 (numérique)

Découvrir la revue

Citer ce compte rendu

Fiola, M. A. (2007). Compte rendu de [Freddie Plassard. Lire pour traduire.

Paris, Les Presses de la Sorbonne Nouvelle, 2007, 323 p.] TTR, 20(2), 327-329.

https://doi.org/10.7202/018829ar

Tous droits réservés (C) TTR: traduction, terminologie, rédaction - Les auteurs, 2008
Ce document est protégé par la loi sur le droit d'auteur. L'utilisation des services d'Érudit (y compris la reproduction) est assujettie à sa politique d'utilisation que vous pouvez consulter en ligne.

https://apropos.erudit.org/fr/usagers/politique-dutilisation/ 


\section{COMPTES RENDUS}

\section{Freddie Plassard. Lire pour traduire. Paris, Les Presses de la Sorbonne Nouvelle, 2007, 323 p.}

L'ouvrage que propose Plassard, issu de ses recherches doctorales, présente un tableau fort complet de la problématique de la lecture dans l'optique de la traduction. Il convient ici effectivement de parler de problématique, car ce sujet, encore peu exploré en traductologie, comporte de multiples facettes. En effet, lorsqu'on songe à la lecture en traduction, on pense d'emblée à la lecture forcément nécessaire qui devance l'étape de la recherche documentaire éventuelle (laquelle passe également par la lecture) et de la compréhension. Toutefois, il ne faut pas oublier l'importance de la lecture à toutes les autres étapes ultérieures, de la réexpression à la vérification. C'est donc à une étude approfondie de la lecture et de la convergence de cette pratique avec l'exercice traductionnel que nous convie Plassard.

$\mathrm{Au}$ fil des sept chapitres qui composent son ouvrage, l'auteure brosse un tableau des divers horizons théoriques entourant, d'une part, la pratique de la lecture et, d'autre part, la pratique de la traduction, et parvient à effectuer des rapprochements théoriques fort justes entre ces deux objets, rapprochements qui passent par une série de questions, autour desquelles est érigée la structure du livre. Ces sept chapitres s'intitulent : 1 . Le pacte de lecture; 2. Le savoir-lire; 3. Mise en œuvre d'un parcours de lecture; 4. La compréhension; 5. L'intertextualité, principe de lecture et d'écriture; 6. La lecture documentaire en traduction; 7. La lecture, outil d'amélioration de la traduction.

Comme on peut en juger, le contrat est ambitieux et la diversité des thèmes abordés dans l'ouvrage témoigne de la complexité du sujet. Or,l'auteure parvient éloquemment à effectuer une synthèse convaincante en tissant des liens entre deux champs de recherche connexes évoluant depuis longtemps en parallèle. Ils sont ici, pour notre plus grand bénéfice, rassemblés, et les points de jonction sont clairement mis en relief. 
Pour décrire cet objet d'étude depuis trop longtemps négligé par les traductologues, l'auteure a ressenti le besoin de se donner un métalangage glané çà et là au fil de ses lectures, entre autres en psychologie cognitive, en théorie littéraire, en histoire et, bien entendu, en traductologie, ce qui lui permet d'articuler son argument d'autant plus clairement et avec rigueur. Les lecteurs qui craindraient de s'y perdre n'ont pas de raison de s'inquiéter, car l'auteure a prévu un glossaire, présenté en fin d'ouvrage, qui jette toute la lumière nécessaire sur les nouvelles dénominations qu'elle cherche ainsi à introduire dans le champ de la traductologie.

D'une page à l'autre, l'auteure reprend certains concepts de la théorie interprétative de la traduction que l'on croyait dorénavant épuisés. Cependant, vus sous la loupe de Plassard et à partir d'une perspective de lecture, certains concepts sont révélés sous un jour nouveau, ce qui ne fait qu'en rehausser la pertinence. Par exemple, à la page 56, on peut lire :

Si les codes et sous-codes doivent être actualisés selon la 'compétence de destination', leur appropriation par le traducteur restera subordonnée à son aptitude à se fondre dans la peau du lecteur modèle construit par le texte original, d'une part, mais tout autant à son aptitude à pouvoir à son tour se positionner comme auteur-modèle du texte ainsi approprié, à destination de nouveaux lecteurs empiriques, de l'autre.

En lisant l'ouvrage de Plassard, il est un type de lecture qui, cependant, brille par son absence : la lecture de l'hypertexte. En fait, si l'auteure traite de l'« hypertexte ", elle l'entend au sens de " texte original» (p. 265) et non dans son sens informatique de système de documents liés entre eux par des hyperliens, permettant de passer automatiquement d'un texte à un autre. Nous souhaiterons donc que, lorsque l'auteure aura l'occasion de se replonger dans son sujet, elle tente de voir dans quelle mesure l'avènement d'Internet et de l'hypertexte a un effet sur les stratégies de lecture et, partant, d'écriture, dans le sens où la lecture linéaire tend à céder de plus en plus le pas à la lecture « en trois dimensions ", où les liens logiques ne sont plus forcément exprimés par les charnières, mais plutôt par ces hypercharnières que sont les liens hypertextes. 
En conclusion, l'ouvrage de Plassard saura très certainement intéresser quiconque a pour préoccupation la pratique de la lecture et de l'écriture, surtout de textes pragmatiques, ce qui ne devrait pas manquer de retenir l'attention des praticiens et, a fortiori, des formateurs des langagiers d'aujourd'hui et de demain. Toutefois, ceux et celles qui s'intéressent également à la didactique des langues ne seront pas en reste.

\section{Marco A. Fiola UnIVERSITÉ RYERSON}

\section{Jean Delisle. La traduction en citations. Ottawa, Presses Universitaires d'Ottawa, 2007, 396 p.}

« Je le dis tout de suite : ce dictionnaire de citations sur les traductions est un régal " (Henri Meschonnic, préface du livre). Voici plus de 3000 aphorismes, éloges, injonctions, opinions, règles, jugements... sur la traduction et les traducteurs et interprètes glanés chez plus de 800 écrivants, de l'Antiquité à 2005, et classés sous une centaine de thèmes.

Le régal tient à la fois à la diversité des sources, des périodes, des points de vue et aux itinéraires que permet un tel répertoire. La promenade est d'abord insouciante et légère, à travers des lieux en apparence communs. Puis assez vite, l'invitation au vagabondage nécessite une boussole pour se repérer, avancer, car le lecteur ressent la diversité, sinon les contradictions qui se pointent : il est appelé ainsi peu à peu à situer son propre horizon, à baliser son propre chemin. Ce qui pouvait apparaître comme un dictionnaire accumulatif, un inventaire de déjà vu, se révèle comme un espace de courants d'air qui décoiffent : les discours sur la traduction et les traducteurs ont tout dit et leur contraire!

La préface d'Henri Meschonnic (pp. XI-XIV) cerne bien les multiples miroirs que nous renvoie ce dictionnaire de clichés et d'anti-clichés - ce « jeu de massacres » qui porte sur le pensé et l'impensé touchant la traduction. Quant à l'avant-propos de Jean Delisle (pp. XV-XXXIV), il déjoue à l'avance les pièges que 